\title{
RE: Geobiology reveals how human kidney stones dissolve in vivo (by: Sivaguru et al. 2018)
}

\author{
Vincent De Coninck ${ }^{1,4,5} \cdot$ Etienne Xavier Keller ${ }^{2,4,5} \cdot$ Michel Daudon $^{3} \cdot$ Olivier Traxer $^{4,5}$ (1)
}

Received: 6 May 2019 / Accepted: 17 May 2019 / Published online: 7 June 2019

๑) Springer-Verlag GmbH Germany, part of Springer Nature 2019

Sivaguru et al. evaluated the evolution of calcium oxalate stones using geobiology [1]. They observed how calcium oxalate stones undergo multiple events of dissolution during crystallization and growth in the kidney based on super-resolution auto-fluorescence images. They concluded that their results "modify the long-held working assumption that calcium oxalate is strongly insoluble in vivo". We disagree with this statement since their findings are nothing new under the sun.

The concept that calcium oxalate monohydrate (COM) stones can be either formed by primary crystallization or by dehydration of calcium oxalate dihydrate (COD) stones has been described more than 50 years ago. In 1962, Murphy and Pyrah described the transition and recrystallization of COD to COM after analysis of stones with aggregates of monohydrate crystals in the centre and dihydrate crystals in the periphery [2]. They reported that the original deposition was COD, which later crystallized to COM. This was illustrated with a picture showing a COD crystal that was partially

Olivier Traxer

olivier.traxer@aphp.fr

Vincent De Coninck

vincent.de.coninck@klina.be

Etienne Xavier Keller

etienne.xavier.keller@gmail.com

Michel Daudon

michel.daudon@aphp.fr

1 Department of Urology, AZ Klina, Brasschaat, Belgium

2 Department of Urology, University Hospital Zurich, University of Zurich, Zurich, Switzerland

3 Laboratoire des Lithiases, Service des Explorations Fonctionnelles Multidisciplinaires, AP-HP, Hôpital Tenon, Paris, France

4 Sorbonne Université, Service d'Urologie, AP-HP, Hôpital Tenon, 75020 Paris, France

5 Sorbonne Université, GRC n²0, Groupe de Recherche Clinique sur la Lithiase Urinaire, Hôpital Tenon,

75020 Paris, France recrystallized to COM. This well-known phenomenon was also described by Cifuentes Delatte et al. in 1972 using polarization microscopy and infrared spectroscopy [3]. They reported the transformation of COD to COM in the centre of stones that became covered by the apposition of new COD layers. They additionally found that crystals in a transition phase did neither have the typical morphological aspects of COD nor $\mathrm{COM}$, but that these crystals clearly corresponded to COM with infrared spectroscopy. After incubation experiments and scanning electron microscopy, Berg et al. found in 1979 that COD crystals dehydrate to COM crystals via dissolution and recrystallization, starting at the corners and edges [4].

This dissolution and conversion process from COD to COM must be taken into account when making relationships between stone morphology, crystalline composition and possible etiopathogenic factors [5]. However, stating that "these observations open a fundamentally new paradigm for clinical approaches that include in vivo stone dissolution" is premature, since this study only confirms research from the last half century.

\section{References}

1. Sivaguru M, Saw JJ, Williams JC Jr, Lieske JC, Krambeck AE, Romero MF et al (2018) Geobiology reveals how human kidney stones dissolve in vivo. Sci Rep 8:13731

2. Murphy BT, Pyrah LN (1962) The composition, structure, and mechanisms of the formation of urinary calculi. Br J Urol 34:129-159

3. Cifuentes Delatte L, Rapado A, Hodgkinson A (1972) Urinary calculi. In: Recent advances in aetiology, stone structure and treatment. Proceedings of the international symposium on renal stone research, Madrid

4. Berg W, Lange P, Bothor C, Rossler D (1979) Submicroscopic investigations on calcium oxalate stone genesis. Eur Urol 5:136-143

5. Daudon M, Bader CA, Jungers P (1993) Urinary calculi: review of classification methods and correlations with etiology. Scan Microsc 7:1081-1104

Publisher's Note Springer Nature remains neutral with regard to jurisdictional claims in published maps and institutional affiliations. 